\title{
Risk Analysis for Unintentional Slide Deployment During Airline Operations
}

\author{
Eduardo S. Ayra, ${ }^{1}$ David Ríos Insua, ${ }^{2, *}$ María Eugenia Castellanos, ${ }^{1}$ and Lydia Larbi ${ }^{3}$
}

\begin{abstract}
We present a risk analysis undertaken to mitigate problems in relation to the unintended deployment of slides under normal operations within a commercial airline. This type of incident entails relevant costs for the airline industry. After assessing the likelihood and severity of its consequences, we conclude that such risks need to be managed. We then evaluate the effectiveness of various countermeasures, describing and justifying the chosen ones. We also discuss several issues faced when implementing and communicating the proposed measures, thus fully illustrating the risk analysis process.
\end{abstract}

KEY WORDS: Aviation safety; Bayesian analysis; cost models; risk analysis; risk communication

\section{INTRODUCTION}

Increasing competition within a shrinking economy is forcing airlines to reduce costs. This might eventually affect aviation safety, in spite of being a critical factor in this industry. Within such complicated context, it is worth noting that risk analyses in this sector have been based on relatively simple methods to identify, assess, and communicate risks. $^{(1)}$ As an example, standard aviation safety procedures tend to promote the use of risk matrices in spite of well-known criticisms. ${ }^{(2)}$ Also, expected loss values are frequently used to summarize risks, even though this may not be adequate to capture the low-probability high-consequence events usually associated with safety-critical systems, as it happens in aviation safety. ${ }^{(3)}$ For this reason, there is a need to develop and improve current procedures and tools to analyze risk factors in air transportation systems. ${ }^{(4-6)}$

We analyze here risks associated with a relevant air transportation incident: the unintended

\footnotetext{
${ }^{1}$ Department of Informatics and Statistics, Universidad Rey Juan Carlos 28933, Mostoles, Spain.

${ }^{2}$ ICMAT-CSIC, 28049 Madrid, Spain.

${ }^{3}$ Department of Mathematics, Universite Tizi Ouzou, Algeria.

*Address correspondence to David Rios Insua, ICMAT-CSIC, 28049 Madrid, Spain; david.rios@icmat.es.
}

deployment of slides under normal operations within a commercial airline. Since the 1960s, manufacturers have been required to develop and install inflatable slides at emergency and exit doors, according to certified standards, which facilitate escaping from the aircraft to the ground level during emergencies. As an example, the Federal Aviation Administration (FAA) requires slides on all aircraft exit doors that are 6 feet or more above ground level, since the evacuation of the entire aircraft should be done in 90 seconds using $50 \%$ of the available evacuation exits. Standard operating procedures establish that cabin crew members must set exit doors in armed (or automatic) mode prior to taxi out for departing. In the event of an emergency, when the door is opened, the slide deploys automatically, providing a means of quickly getting out of the aircraft.

Many of such slides are unintentionally deployed under normal operations every year all over the world. This has actually become a global safety issue for passengers and handling staff, with relevant financial implications. An IATA report ${ }^{(7)}$ outlined this problem from an economical point of view, estimating an associated cost of around 20M USD per year across the airline industry.

We provide a formal risk analysis for this problem; there are several papers with methodological 


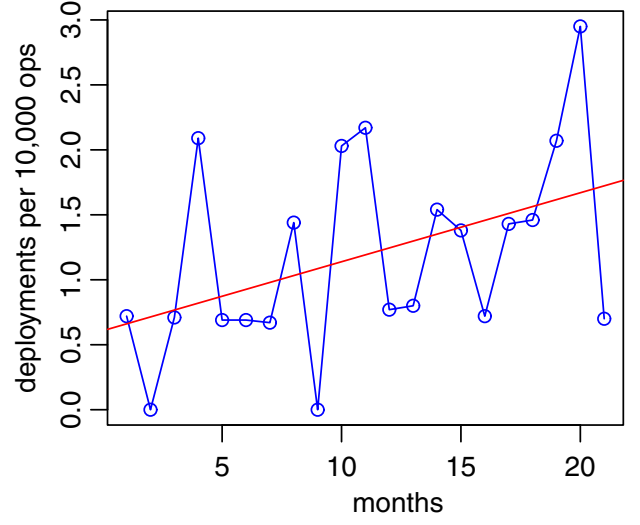

Fig. 1. Monthly deployments per 10,000 ops and trend over 21 months.

introductions. ${ }^{(8,9)}$ Our analysis is based on historical recorded data as much as possible, relying on expert opinion to fill the gaps whenever data are not available. For that purpose, we draw on rigorous elicitation methods. ${ }^{(10,11)}$

We first assess the entailed risks, providing a model for the occurrence of such incidents and their associated costs, and combine them to provide forecasts for the corresponding total costs. As these are deemed too high, we consider possible countermeasures and determine the most effective ones. We conclude with some discussion concerning issues in relation to the implementation of such countermeasures and communicating the entailed risks, therefore illustrating all the phases of a risk analysis.

\section{RISK ASSESSMENT}

We start by assessing risks in relation to unintended slide deployment under normal operations in a main commercial airline, within the classic risk analysis framework. ${ }^{(12)}$ An exploratory analysis of monthly incident rates over 21 months of operations shows an increasing trend, suggesting the growing relevance of this problem, see Fig. 1.

\subsection{Incident Data Analysis}

We start by making inferences about the occurrence rate of this type of incident, as well as forecasts of the number of incidents. A maximum likelihood estimate for the incident proportion for the company, given the available data, is 0.00011 , for 35 incidents over 304,514 operations, or approximately 1.1 incidents per 10,000 operations.
Table I. Testing Factors Potentially Affecting Incidents

\begin{tabular}{lll}
\hline Factor & \multicolumn{1}{c}{ Relevance } & \multicolumn{1}{c}{ Factor Levels } \\
\hline Aircraft type & Yes (Moderate) & A $>$ B \\
Airport & No & Nearly 50 \\
Pairing day & Yes (Moderate) & First $>$ Second $>$ Third \\
Flight turn & Yes (Moderate) & First $>$ ( Second, Third) \\
\hline
\end{tabular}

Through discussion with experts and a literature review, we identified four factors potentially affecting the unintended deployment of slides under normal operations: the type of aircraft; the airport; the pairing day; and, fourthly, the flight turn within the corresponding pairing day. We performed equal proportion tests in relation to those factors, based on a beta-binomial model (for two proportions) or a Dirichlet-multinomial model (for several proportions) with noninformative priors; see Ref. 13 for details. Table I summarizes the obtained results.

As an example, as far as the aircraft type is concerned, the data available are 30,355 operations, with seven incidents, for type A aircrafts, and 262,825 operations, with 28 incidents, for type B aircrafts. A $90 \%$ credible interval for the difference of incident proportions between type A and B airplanes is $[0.000011,0.0003]$, which suggests a slightly bigger incident proportion for type A planes.

Thus, we believe that the following factors might have influence over the proportion of incidents.

- Aircraft type. As mentioned, there seems to be a slightly bigger proportion of incidents for the A fleet, for which most flights are long haul, than for the B fleet, whose flights tend to be short haul. This might be due to a slightly poorer situational awareness of crew at longhaul flights.

- Pairing day. Considering a typical three-day onduty pairing, it seems that there are slightly more incidents on the first day than on the second one, and on the second one than, in turn, on the third day of a pairing. This might be due to poor situational awareness of crew after a few days off, which gradually increases flight after flight, especially on long-haul flights.

- Flight turns. We have considered the usual three flights per day on short-haul flights, and one flight per day, on long-haul ones. In this context, there seems to be a slightly bigger proportion of incidents in the first turn. For the second and third turns in short-haul flights, the 
Table II. Data Concerning Factors Potentially Affecting Incidents

\begin{tabular}{lcclc}
\hline $\begin{array}{l}\text { Case } \\
i\end{array}$ & $\begin{array}{c}\text { Operations } \\
n_{i}\end{array}$ & $\begin{array}{c}\text { Incidents } \\
y_{i}\end{array}$ & $\begin{array}{c}\text { Factor Lvls. } \\
\text { (Fleet,Day,Turn) }\end{array}$ & $\begin{array}{c}\text { Coding } \\
x_{i}\end{array}$ \\
\hline 1 & 29,702 & 3 & B,Fst,1 & $1,1,1$ \\
2 & 59,661 & 7 & B,Fst,Oth & $1,1,2$ \\
3 & 44,159 & 6 & B,Snd,1 & $1,2,1$ \\
4 & 46,257 & 6 & B,Snd,Oth & $1,2,2$ \\
5 & 28,910 & 2 & B,Thrd,1 & $1,3,1$ \\
6 & 55,193 & 4 & B,Thrd,Oth & $1,3,2$ \\
7 & 15,245 & 6 & A,Fst,1 & $2,1,1$ \\
8 & 1,516 & 0 & A,Fst,Oth & $2,1,2$ \\
9 & 13,713 & 1 & A,Thrd,1 & $2,3,1$ \\
\hline
\end{tabular}

proportions seem similar. On long-haul flights, poor situational awareness could provide an explanation. On short-haul flights, time pressure to be on schedule, plus connecting passengers pressing on cockpit and cabin crew members to leave the aircraft as soon as possible so as to reach connecting flights, could be a contributing factor to the incident, because of the distractions they entail.

As a consequence, we have fitted a binomial logistic regression model to the incident proportions with:

$$
\begin{gathered}
\left(x_{i}, n_{i}, y_{i}\right), i=1, \ldots, k \\
y_{i} \mid \theta_{i} \sim \operatorname{Bin}\left(n_{i}, \theta_{i}\right), \\
\operatorname{logit}\left(\theta_{i}\right)=\alpha+\beta x_{i},
\end{gathered}
$$

where $i$ designates the factor level, $x_{i}$ the corresponding factor level values, $n_{i}$ the number of operations under such conditions, and $y_{i}$ the number of observed incidents. The parameters of the model are the $\theta_{i} \mathbf{s}$, which are the proportion of incidents under level $i$, and depend on the parameters $\alpha$ and $\beta$. We have identified $k=9$ relevant levels, as described in Table II, which provides the data used to estimate the model. Note that the fleet factor has two values (A, B). Therefore, we estimate one $\beta$ parameter for it. The pairing day has three factor levels (Fst, Snd, Thrd); thus, we need to estimate two $\beta$ parameters for it. Finally, flight turn has two values (1, Oth), therefore requiring just one $\beta$ parameter.

We use vague normal priors (with mean 0 and precision $10^{-4}$ ) for all the parameters, adopting the strategy in several works. ${ }^{(14,15)}$ Essentially, our Markov chain Monte Carlo (MCMC) sampler leads
Table III. Phase and Staff Potentially Affecting Incidents

\begin{tabular}{lc}
\hline Factor & Relevance Ranking \\
\hline Operational phase & $\begin{array}{c}\text { Arrival }>\text { Departure }>>\text { Refueling } \\
(\text { Turnround })>\text { Preflight (Stopover }) \\
(\mathrm{a}, \mathrm{b})>(\mathrm{c}, \mathrm{d}, \mathrm{e}, \mathrm{f}, \mathrm{g}, \mathrm{h}, \mathrm{i})\end{array}$ \\
\hline
\end{tabular}

Table IV. Slide Costs (In-House and External) Depending on Affected Gate and Aircraft

\begin{tabular}{cccccc}
\hline & A1 & A2 & A3 & A6 & A6w \\
\hline$C_{m}^{i}$ & 4,160 & 4,040 & 2,400 & 3,630 & 3,210 \\
$C_{m}^{e}$ & 6,429 & 4,850 & 5,785 & 7,423 & 4,946 \\
\hline & $\mathrm{Bf}$ & & $\mathrm{Ba}$ & $\mathrm{Bw}$ & $\mathrm{B} 2 / 3$ \\
\hline$C_{m}^{i}$ & 1,840 & & 1,480 & 1,630 & 1,430 \\
$C_{m}^{e}$ & 2,605 & & 2,323 & 4,571 & 4,741 \\
\hline
\end{tabular}

to posteriors centered on the classic logistic regression estimates with some uncertainty around them.

The posterior means were -9.03 for $\alpha$ and $(0.83$, $0.06,-0.77,-0.03)$ for the $\beta$ parameters, with corresponding standard deviations $(0.39,0.52,0.41,0.46$, 0.38 ). Only $\alpha$ and the first $\beta$ parameter, corresponding to the fleet type, seemed a posteriori relevant, in the sense of being different from 0 . They were the only parameters finally retained in the model, effectively leaving two levels (fleet A and B). The posterior means were -9.18 for $\alpha$ and 0.74 for $\beta_{1}$, and the respective standard deviations were 0.19 and 0.42 .

Model fit was evaluated in two ways. First, as a quick diagnostic, based on the classic estimators, the residual deviance (5.896 with 7 degrees of freedom) was smaller than the null deviance ( 8.739 with 8 d.f.) with $p$-value of 0.55 and AIC (35.5) smaller than that of the full model (37.9). Second, we built predictive intervals for the number of deployments for the nine cases. For example, the 0.8 equal tail predictive probability interval for case 4 was $[4,14]$ for six observed incidents. In all cases, we covered the observations, concluding that our model had good predictive properties.

With a view toward risk management, we also studied in which operational flight phase the incident occurred, and who could be considered responsible for it, with results described in Table III, based on multinomial-Dirichlet models. ${ }^{(16)}$ 
This suggests that:

- Most incidents take place during arrival, rather than when departing, than in the other operational phases. This might occur because flight and cabin crew are distracted and pushed by passengers in a hurry to get out of the aircraft upon arrival.

- Most incidents seem to be caused by type (a, b) personnel, who tend to operate closer to the slides.

Also, with a view toward risk management, we analyzed the reported causes of incidents based on the framework introduced in a previous work, ${ }^{(12)}$ which classifies into errors (decision, skill-based, or perceptual errors) and violations (routine or exceptional). All causes of incidents reported within the database can be described as skill-based errors: 7 inadvertent use of handles (door or arm/disarm), 9 failures in prioritizing attention (procedure interruptions), and 19 omitted steps in procedure (procedure noncompliances). These suggest some incidents caused by inattention and numerous incidents probably due to current inadequate procedures. A posteriori, we model the proportions of error types through a Dirichlet distribution with parameters 8, 10, and 20.

\subsection{Modeling Incident Costs}

Unintended slide deployment could potentially cause serious, even fatal, injuries to crew and ground staff working in the vicinity of the aircraft. However, we have concentrated on economic impacts, mainly due to lack of reported data concerning injuries over the period of interest, and, we could say, even over the whole history of the incumbent airline.

As there are so many variables involved in this type of incident, actual costs become difficult to estimate. However, for major airlines, the key costs refer mainly to repairing the slide system and to the potential delays originated by the incident. Thus, we shall take into account the following cost components: the cost of repairing the slide, which comprises (i) the maintenance crew labor costs to remove the deployed slide and install a suitable one, when applicable, which essentially depends on the time $T_{m}$ required for disassembly and assembly, (ii) the transportation costs of the slide, $C_{t}$, and (iii) the maintenance work costs, $C_{m}$, which will depend on the type of aircraft and emergency exit affected, and whether the company repairs the slide in-house or at an external workshop; and the ground delay associated costs, described as the induced delay $T_{d}$ times the cost $C_{d}$ per time unit associated with the delay. As a consequence, the incurred costs $C$ (in euros) per incident are modeled through the random variable:

$$
C=C_{l} \times T_{m}+C_{t}+C_{m}+C_{d} \times T_{d},
$$

where $C_{l}$ represents the labor maintenance costs. We describe in detail the component costs used in our analysis.

The maintenance labor cost $C_{l}$ is 65 euros/hour per technician. With regard to $T_{m}$, we use judgmental elicitation using the methodology in Galway. ${ }^{(17)}$ An expert provided assessments for the minimum (30 minutes), maximum (60 minutes), and most likely (45 minutes) work durations. To mitigate expert overconfidence, we assume a triangular distribution with 0.05 and 0.95 quantiles over the minimum and the maximum, leading to a triangular distribution with parameters $(0.385,0.75,1.115)$, which are measured in hours.

For $C_{t}$, we use judgmental elicitation as before. We asked an expert to provide assessments for the minimum (60 euros), maximum (180 euros), and most likely (110 euros) transportation costs. We assess it as a triangular distribution with parameters $(34,110,210)$, again mitigating expert overconfidence.

$C_{m}$ is described through the mixture:

$$
C_{m}=q \times C_{m}^{i}+(1-q) \times C_{m}^{e},
$$

where $q$ represents the proportion of slides repaired in-house and superscripts $i$ and $e$ refer to in-house and external maintenance workshops, respectively.

For $q$, we used judgmental elicitation with an expert. He assessed that around $80 \%$ of the slides are repaired externally, expressing little uncertainty about such assessment. As a consequence, after checking for consistency, we assessed a beta distribution with parameters $(16,4)$, with mean 0.8 and standard deviation 0.08 .

The costs $C_{m}^{i}$ and $C_{m}^{e}$ will depend on the type of slide repaired, expressed in Table IV, for various types of doors in aircrafts A and B, respectively.

We have observed the following incidents for each type of door, which leads us to the following Dirichlet posterior parameters under a vague prior, with separate models for A and B aircraft types (see Tables V and VI).

Note that the A1 and Bf gates, which are the most affected by this type of incident, are attended by type $\mathrm{a} / \mathrm{b}$ personnel, as highlighted above. 
Table V. Incidents and Posterior Parameters for A Aircraft-Type Doors

\begin{tabular}{lccccc}
\hline & A1 & A2 & A3 & A6 & A6w \\
\hline Incidents & 4 & 2 & 1 & 0 & 0 \\
Parameters & 5 & 3 & 2 & 1 & 1 \\
\hline
\end{tabular}

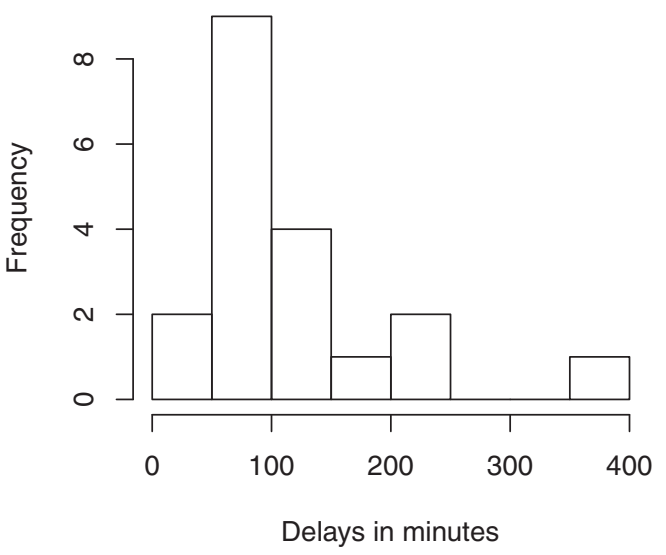

Fig. 2. Nonzero and nonextreme delays in minutes for incidents.

We deal now with delays $T_{d}$ for the recorded incidents. Out of 35 events, 13 incidents entailed no delay, or the delay was actually absorbed by the route network. For the remaining 22 times, we observed 20 standard nonzero delays and two extremely long delays, of approximately 15 and 20 hours, associated with A type aircrafts departing from a specific airport. We provide the histogram of zero and nonextreme data in Fig. 2.

Therefore, we decomposed the delay $T_{d}$ through the mixture:

$$
\begin{gathered}
T_{d}=p_{0} I_{0}+p_{1} F_{d} \\
p_{0}+p_{1}=1, \\
p_{0}, p_{1} \geq 0,
\end{gathered}
$$

where $p_{0}$ represents the proportion of incidents that do not entail a delay; $I_{0}$ denotes the indicator function at 0 ; and, finally, $F_{d}$ describes nonzero delays. Through a standard noninformative beta-binomial model, we get:

$$
p_{0} \mid \text { data } \sim B e(14,23) .
$$

We model nonzero delays, extreme and nonextreme ones, considering separately the delays for types A $\left(F_{d_{A}}\right)$ and $\mathrm{B}\left(F_{d_{B}}\right)$ aircrafts. Indeed, we represent:

$$
F_{d_{A}} \sim p \operatorname{Wei}(\theta=0, \alpha, \beta)+(1-p) \operatorname{Wei}(\theta, \alpha, \beta),
$$

Table VI. Incidents and Posterior Parameters for B Aircraft-Type Doors

\begin{tabular}{lcccc}
\hline & $\mathrm{Bf}$ & $\mathrm{Ba}$ & $\mathrm{Bw}$ & $\mathrm{B} 2 / 3$ \\
\hline Incidents & 17 & 4 & 1 & 5 \\
Parameters & 18 & 5 & 2 & 6 \\
\hline
\end{tabular}

Table VII. Incurred Delay Costs per Minute for A and B Flights (Values in Parentheses Are Minimum, Most Likely, and Maximum Costs for Each Combination)

\begin{tabular}{lcc}
\hline & A & B \\
\hline Passenger hard & $(0.12,0.19,0.24)$ & $(0.12,0.19,0.24)$ \\
Passenger soft & $(0.06,0.19,0.22)$ & $(0.06,0.19,0.22)$ \\
Marginal crew & $(0.00,14.00,39.00)$ & $(0.00,7.90,16,59)$ \\
Marginal maintenance & $(0.65,0.81,0.97)$ & $(0.38,0.47,0.56)$ \\
\hline
\end{tabular}

Table VIII. Costs Associated with an A Incident for Aircraft Types A and B

\begin{tabular}{lcc}
\hline & Type A & Type B \\
\hline Mean & 10,970 & 2,959 \\
Median & 6,808 & 2,590 \\
$Q_{0.95}$ & 30,481 & 5,503 \\
$Q_{0.98}$ & 35,189 & 6,129 \\
\hline
\end{tabular}

Table IX. Countermeasure Incident Reduction and Cost

\begin{tabular}{lccccc}
\hline & \multicolumn{5}{c}{ Error Noncomp. Interr. Cost/u. Total Cost } \\
\hline $\begin{array}{l}\text { Procedure revision } \\
\text { Awareness }\end{array}$ & 75 & 0 & 95 & 0 & 0 \\
$\quad$ campaign & 30 & 30 & 30 & 7,000 & 7,000 \\
$\quad \begin{array}{l}\text { Warning devices, } \\
\quad \text { strategy 1 }\end{array}$ & 99 & 99 & 99 & 1,800 & $1,300,000$ \\
$\quad \begin{array}{l}\text { Warning devices, } \\
\quad \text { strategy 2 }\end{array}$ & 99 & 99 & 99 & 1,800 & 216,000 \\
$\begin{array}{l}\text { Visual reminders, } \\
\quad \text { strategy 1 }\end{array}$ & 20 & 20 & 20 & 55 & 40,000 \\
$\quad \begin{array}{l}\text { Visual reminders, } \\
\quad \text { strategy 2 }\end{array}$ & 20 & 20 & 20 & 55 & 6,600 \\
\hline
\end{tabular}

$$
F_{d_{B}} \sim \operatorname{Wei}(\theta=0, \alpha, \beta),
$$

where $\operatorname{Wei}(\theta, \alpha, \beta)$ designates the Weibull density with location parameter $\theta$, scale parameter $\alpha$, and shape parameter $\beta$, with parameterization:

$$
f(x \mid \theta, \alpha, \beta)=\alpha \frac{(x-\theta)^{\alpha-1}}{\beta^{\alpha}} \exp \left(-((x-\theta) / \beta)^{\alpha}\right) .
$$

We use reference priors for the scale and shape Weibull parameters. ${ }^{(18)}$ For $p$, we have considered 
Table X. Expected Net Present Costs for One and Five Years of Operations

\begin{tabular}{lcc}
\hline Countermeasure & One Year & Five Years \\
\hline Procedure revision & 252,902 & $1,214,935$ \\
Awareness campaign & 524,477 & $2,492,943$ \\
Warning devices, strategy 1 & $1,307,393$ & $1,335,514$ \\
Warning devices, strategy 2 & 616,058 & $2,137,866$ \\
Visual reminders, strategy 1 & 631,403 & $2,881,078$ \\
Visual reminders, strategy 2 & 677,329 & $3,228,759$ \\
None & 663,400 & $2,224,520$ \\
\hline
\end{tabular}

Table XI. Countermeasure Effectiveness After Procedure Revision Has Been Implemented

\begin{tabular}{lccc}
\hline & Error & Noncomp. & Interr. \\
\hline Awareness campaign & 0 & 70 & 0 \\
Warning devices, strategy 1 & 99 & 99 & 99 \\
Warning devices, strategy 2 & 99 & 99 & 99 \\
Visual reminders, strategy 1 & 0 & 10 & 0 \\
Visual reminders, strategy 2 & 0 & 10 & 0 \\
\hline
\end{tabular}

Table XII. Expected Net Present Costs for Countermeasures for One and Five Years in Addition to Procedure Revision

\begin{tabular}{lcc}
\hline Countermeasure & One Year & Five Years \\
\hline Awareness campaign & 123,724 & 567,739 \\
Warning devices, strategy 1 & $1,302,529$ & $1,312,149$ \\
Warning devices, strategy 2 & 352,862 & 873,480 \\
Visual reminders, strategy 1 & 273,448 & $1,161,478$ \\
Visual reminders, strategy 2 & 236,060 & $1,108,918$ \\
None & 252,902 & $1,214,935$ \\
\hline
\end{tabular}

a beta prior with mean 0.75 and standard deviation 0.22 , whereas for the location parameter $\theta$ in the Weibull component of the mixture for extreme data, we have used a uniform distribution between 11 and 16 hours. We used a Metropolis within Gibbs sampling scheme, with 50,000 iterations after a burnin period of 30,000 , to simulate from the posteriors with adequate model fit, as shown in Fig. 3.

As for the delay costs $C_{d}$, we use a model based on a study by Cook and Tanner. ${ }^{(19)}$ After consulting with company experts, we considered three main cost components: costs related with passenger delay, marginal crew costs, and marginal maintenance costs. As established by Cook and co-authors, total costs integrate a passenger component, which is split into "hard" (related with rebooking, compensation care, etc.) and "soft" (in relation to perceiving the airline as unpunctual and later choosing, consequently, another one) costs. Marginal crew costs refer to the cost of crewing for additional minutes over and above those planned, whereas marginal maintenance costs entail factors such as the mechanical attrition of an aircraft waiting at gates. We have adopted models for long- and short-haul flights based on several works, ${ }^{(19-21)}$ data, and models with minimum, most likely, and maximum values at both scenarios. After updating figures for inflation, we have the costs in euros per minute reflected in Table VII. $C_{d}$ will be the addition of the four delay cost components.

Once having models for all cost components, we drew 10,000 observations from the distribution of incurred costs $C$, as in Equation (2), by sampling from its constituents and adding them up, leading to a predictive cost distribution per incident sample. At this point, it is relevant to question the possible dependence of the four cost components, since it may affect the basic sampling approach described, as well as the estimated dispersion of $C$. Qualitatively, we do not expect dependence between such constituents, except for two reasons:

- The possible positive dependence between $T_{m}$ (disassembly) and $T_{d}$ (delay) times. A large $T_{m}$ might induce passengers waiting for long before taking off and, consequently, losing connections, etc., therefore increasing $T_{d}$. Indeed, we could consider that $T_{d}=T_{m}+X$ for a certain random variable $X$. However, because of industry pressure, $T_{m}$ tends to be performed quite rapidly, not dominating $T_{d}$. Note, anyhow, that we do not have data available for estimating $T_{m}$, estimated through expert judgments.

- This points out the other possible source of dependence, one due to the variables obtained through expert judgment $\left(T_{m}, C_{t}, q\right.$ within $\left.C_{m}\right)$ : an expert could show similar biases in different assessments and hence induce positive correlation. However, we used different experts for different cost constituents and, by this, hope to have mitigated this issue.

Fig. 4 and Table VIII summarize the predictive cost distribution sample for both types of aircrafts, A and $\mathrm{B}$.

As we may see, the costs per incident can be quite considerable, specially for type A flights, potentially vanishing benefits from an operation affected by a deployment. Fig. 5 provides the contribution of the four component costs to the deployment-induced distribution. Note that this is dominated by the delayrelated costs, and then by the maintenance work costs for both types of flights. 
Delay Time for B Type Aircraft

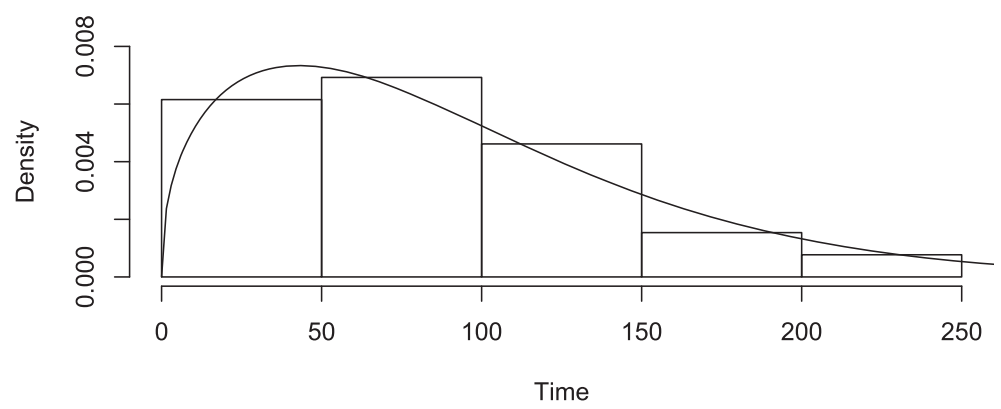

Delay Time for A Type Aircraft

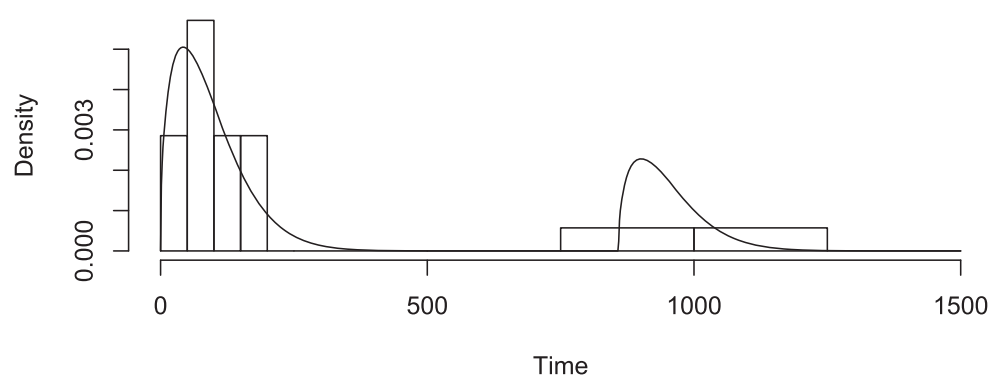

Fig. 3. Delay time histograms, in minutes, for A and $\mathrm{B}$ aircrafts and adjusted model using the posterior mean (continuous line).
We assess now the annual costs associated with the incident for the incumbent airline.

\subsection{Risks Associated with Slide Deployment}

We combine, through simulation, our likelihood and consequence models to complete the risk assessment. In a current typical year, the incumbent airline undertakes around 175,000 operations, out of which 17,000 are of type A and 158,000 are of type B. To simulate the incident costs for one year of operations, for each operation, we simulate whether there is an incident or not; if so, we simulate from the corresponding incident cost-predictive distribution.

Based on 1,000 replications of one-year operations, reflected in Fig. 6, the average annual cost due to incidents is 663,400 euros, whereas the median cost is 655,200 euros. The 0.95 and 0.98 quantiles are, respectively, 836,863 and 893,314 euros. In the current economical setting for the incumbent airline, this is certainly a nonnegligible cost that needs to be managed.

Fig. 7 provides the annual cost components due to the incident for the nine initial flight cases in Table II. Note that A flights are the major contributors to the airline costs in relation to slide

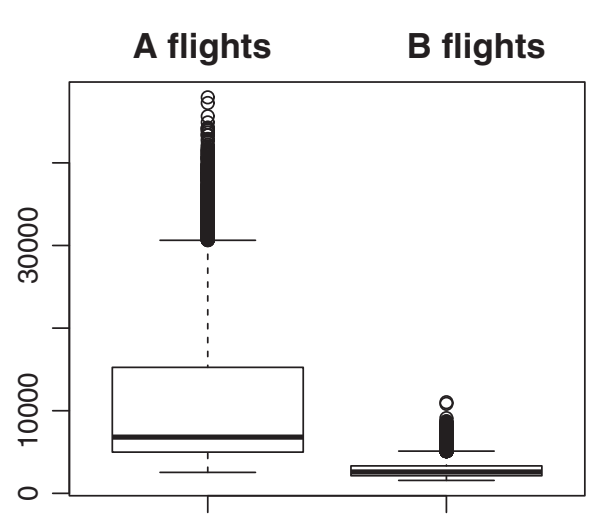

Fig. 4. Distribution costs per incident for types A and B flights.

deployment. They are less used, but the corresponding incidents are more costly when they happen.

\section{RISK MANAGEMENT}

We have identified and analyzed the undesirable effects of unintentional slide deployment. We describe now several relevant countermeasures aimed at mitigating this hazard to an acceptable level for the incumbent company. Thus, we face how to manage the risks associated with this incident. 
Fig. 5. Cost components for types A and B flights.

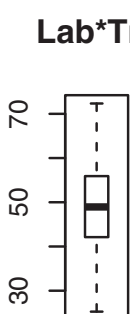

A flights

Lab*Tm

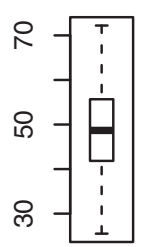

B flights
Transport C

reparation $\mathbf{C}$

delay C

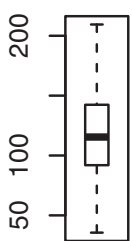

A flights
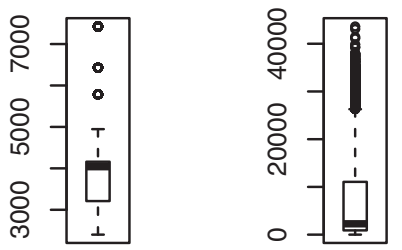

A flights

A flights reparation $\mathbf{C}$

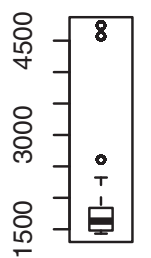

B flights

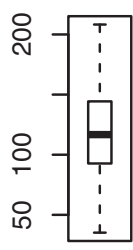

B flights delay C

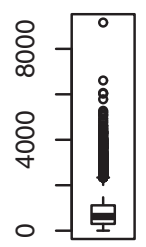

B flights

\subsection{Available Countermeasures}

Based on our logistic regression model (1), the tests performed, a literature review, and brainstorming sessions with airline experts, we devised the following countermeasures.

\subsubsection{Countermeasure 1: Procedure Revision}

A first possibility is the revision of current procedures to avoid interruptions, based on comparisons with procedures and rules of other companies at a similar business level. As an example, at departure, the incumbent company procedure established that flight attendants must arm slides prior to pushback when the aircraft is still with parking brakes set, once doors are closed and the jetway is moved away from the aircraft. Other companies arm slides just prior to taxi, after engine start-up. Similarly, upon arrival, the incumbent company procedure establishes disarming slides after engine shutdown in parking position. Other companies disarm slides just before reaching such stage, while the aircraft is still taxiing in.

This countermeasure tries to separate on time opening/closing and slide arming/disarming procedures and would practically eliminate all incidents due to errors and interrupted procedures, whereas it would have virtually no effect upon other incident causes (noncompliances). It entails nearly no cost, as it is based on manuals and courses that need to be implemented anyway.

\subsubsection{Countermeasure 2: Training Courses and Awareness Campaign}

A second possibility would be to enhance training course contents to alert about the problem, again with almost no cost as both flight and cabin crew have to take part periodically in such courses. This should be coordinated with an awareness campaign, specially for type $\mathrm{a} / \mathrm{b}$ personnel, based on flight safety newsletters and reports on flight safety journals. The information provided in such courses should pay special attention to the identified risk, the severity, and likelihood of its occurrence, explain the actions taken and planned changes, and feed the results of the proposed action back to the company. These countermeasures would have a similar moderate effect on all reported causes of incidents, with an estimated cost of 7,000 euros per year. 


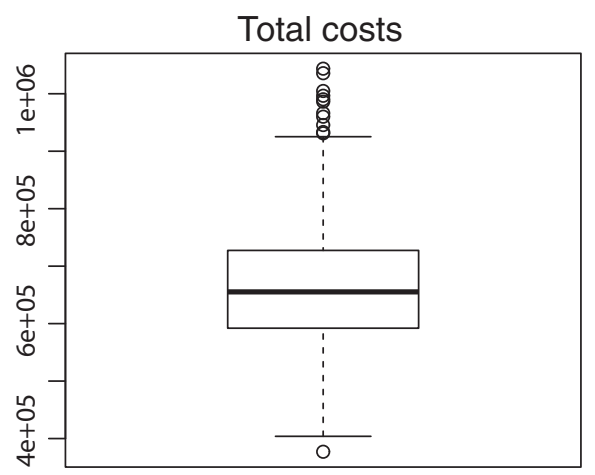

Fig. 6. Annual costs due to incident for a commercial airline.

\subsubsection{Countermeasure 3: Warning Devices}

We could implement aural and visual warning devices that advise when the slide is still in armed or automatic mode and opening the exit door entails a danger. Indeed, recently, some infrared sensor detecting movement devices have been tested in several major airlines, with an estimated installation cost of about 1,800 euros per door. The manufacturer declares that these devices would eliminate practically all incidents. However, its reliability is difficult to set as this system is not yet standard in the industry. If we consider a 120-aircraft fleet with 25 wide bodies (A aircraft type) and 95 narrow bodies (B aircraft type), it would be necessary to install up to 728 devices. This entails around 1,300,000 euros. Apart from the investment, a long time would be needed to implement such countermeasure at the whole fleet. Thus, we could consider implementing them in all gates (strategy 1) or only in gates Bf and A1 (strategy 2), the most affected by slide deployment (see Tables VI and VI). In this second case, the costs have been estimated at 216,000 euros.

\subsubsection{Countermeasure 4: Visual Reminders}

Finally, we could introduce visual reminders close to the operating mechanism with simple messages such as Check slide mode before opening door, with an approximate cost of 55 euros per door. Because of the costs, we could consider two scenarios as before, checking whether they are implemented in all gates (strategy 1 ) or only in gates Bf and A1 (strategy 2). Note, however, that these devices tend to be fairly effective in the beginning, but not that much in the medium term, when people get used to seeing them. Should we implement this countermeasure in all doors and emergency exits, the estimated costs would be around 40,000 euros. Strategy 2 would entail 6,600 euros.

\subsubsection{Countermeasure Summary}

Table IX summarizes the effects of these countermeasures in the reduction of the reported causes of incidents, based on expert opinion, implementation cost per unit, and total implementation cost for the company. For example, the proposed procedure revision would have negligible costs, would reduce errors by $75 \%$, would not practically help in reducing noncompliances, and, finally, would reduce interruptions by $95 \%$.

\subsection{Selection of Optimal Countermeasures}

We describe now how to select the most effective countermeasure. Note that in our case, direct costs associated with incidents will not be affected by their implementation. Thus, we may only aim at reducing incident likelihood through our measures, as described in Table IX, and hope to compensate the costs entailed by their implementation with a reduction in the number of incidents and, consequently, the corresponding savings.

Since the costs involved are small compared with the airline budget, we assume risk neutrality with respect to the involved amounts. ${ }^{(13)}$ Thus, we aim at minimizing expected costs (incident plus implementation) to compare countermeasures. Based on the incident cost model (Equation (2)) and taking into account the annual 17,000 $A$ and $158,000 B$ operations, the general expression for the expected costs per year when we implement countermeasure $M$ is:

$$
\begin{aligned}
& 17,000 \times\left(1-q_{A}^{M}\right) \times\left[C_{l} \times E\left(T_{m}\right)+E\left(C_{t}\right)\right. \\
& +\left[E(q) \times E\left(C_{m A}^{i}\right)+(1-E(q)) \times E\left(C_{m A}^{e}\right)\right] \\
& \left.+E\left(C_{d}^{A}\right) \times E\left(T_{d}^{A}\right)\right]+158,000 \times\left(1-q_{B}^{M}\right) \times\left[C_{l}\right. \\
& \times E\left(T_{m}\right)+E\left(C_{t}\right)+\left[E(q) \times E\left(C_{m B}^{i}\right)\right. \\
& \left.\left.+(1-E(q)) \times E\left(C_{m B}^{e}\right)\right]+E\left(C_{d}^{B}\right) \times E\left(T_{d}^{B}\right)\right]+C^{M},
\end{aligned}
$$

where $C^{M}$ is the implementation cost of the countermeasure, and $q_{A}^{M}$ and $q_{B}^{M}$ are, respectively, the proportion of $A$ and $B$ flights in which the incident does not happen when $M$ is implemented. Similar expressions hold when we consider several-year 
(a)

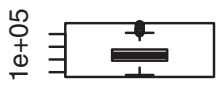

A flights $(2,1,1)$

(a)

$\circ$

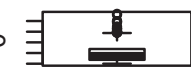

B flights $(1,1,1)$

(d)

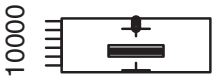

B flights $(1,2,2)$ (b)

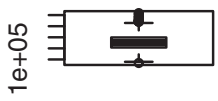

A flights $(2,1,2)$

(b)

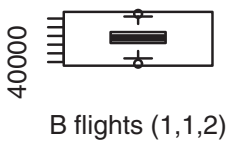

(e)

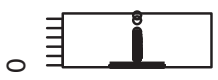

B flights $(1,3,1)$ (c)

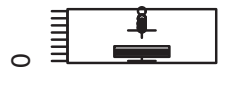

A flights $(2,3,1)$

(c)

○

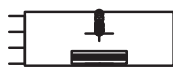

B flights $(1,2,1)$

(f)

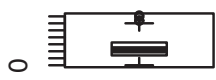

B flights $(1,3,2)$ operation spans. Simple computations lead to the expected cost:

$$
\begin{aligned}
& 17,000 \times\left(1-q_{A}^{M}\right) \times 1,0501.14+158,000 \\
& \times\left(1-q_{B}^{M}\right) \times 4,378.01+C^{M}=17,852,380 \times \\
& \quad\left(1-q_{A}^{M}\right)+691,739,800 \times\left(1-q_{B}^{M}\right)+C^{M} .
\end{aligned}
$$

Table X summarizes the expected net present costs for one and five years of operations with discount factor 0.98 .

Therefore, the most effective countermeasure seems to be the revision of the current procedure. Note, however, that after five years, the strong investment in warning devices might start to pay off, but it is hard to justify such large investment in the current economical context.

We might consider the implementation of several countermeasures simultaneously in addition to procedure revision. Table XI contains the additional effectiveness of countermeasures, on top of that due to procedure revision, as expressed by an expert.

The expected net present costs due to the managed slide deployment for one and five years would be as in Table XII.

Note now that the most effective additional measure would be the introduction of an awareness campaign. All in all, in five years, we are able to reduce expected costs from 2.3 million euros to 0.6 million euros with simple, yet effective, measures.

We conclude with some comments concerning sensitivity analysis. As we mentioned earlier, our operator is risk neutral, for the amounts involved, and we just minimize expected costs, thus being only essentially influenced by the cost component expected values. Fig. 5 suggests that costs are dominated by the delay-induced costs, modeled as $T_{d} \times$ $C_{d}$. With $T_{d}$, there is not much sensitivity analysis performance except for varying the priors. The predictive performance is good enough and pretty robust. With $C_{d}$, it seems advisable to perform sensitivity analysis. The most influential components (and indeed the ones for which there is more uncertainty) are the marginal crew costs; see Table VII. We varied the mode in a neighborhood of the most likely values $(14,7.9)$. However, all countermeasures seemed equally affected, i.e., the countermeasure ranking remained the same, although the expected costs were modified. This suggests robustness of the optimal portfolio of countermeasures (new procedure and campaign) proposed.

\section{DISCUSSION}

Unintentional slide deployment is a main cabin safety issue that has nonnegligible costs for commercial airlines, specially within a context of increasing competition and emphasis on cost consciousness. As an example of its relevance, a manufacturer has developed an innovative audible alert system that could considerably mitigate such threat by warning crew when an exit or emergency door is open in case it should not be (countermeasure 3 ). 
We have provided a detailed risk analysis for such problem for a major airline, assessing the factors that could affect this event and identifying potential countermeasures. Some of them were as simple conceptually as a procedure revision or a risk awareness campaign, yet providing simple and cost-effective solutions.

The company has indeed implemented the proposed countermeasures. In spite of this type of technical studies, implementing the actual decision was not simple because of the usual resistance to change. The company actually had to undertake a communication campaign at various decisionmaking levels, including managers, instructors, and other airline professionals, to make the company aware of the current and potential effects of this incident. Such documents translated the incident and its entailed consequences into a nontechnical language with which decisionmakers could relate, understand, and incorporate into their decisions. We followed the suggestions by ICAO, ${ }^{(22)}$ addressing stakeholders in a targeted way: the management was informed about the potential losses to the organization; crew members involved in implementing the new procedures were informed about the hazards and rationale for the action being taken; and personnel type $a / b$ was specially informed about the severity and likelihood of occurrence of the hazard. Indeed, after a period of six months elapsed since that decision was implemented, the number of deployments has been 4 versus 18 to 24 expected, had the company not implemented the suggested changes. This entailed significant savings, given the current narrow profit margins in the aviation industry.

There are many other relevant risks, specially in the same flight phase (aircraft boarding and loading and taxiing), which might benefit from an approach similar to the one described here. Though only $0.1 \%$ of aviation fatalities occur during these flight phases, statistics indicate that around $8 \%$ of accidents occur during them. An example of this is ground damage events in which an aircraft is damaged by ground equipment. Improving modeling, assessment, management, and communication of risks in the aviation industry will not only enhance safety levels, but will also help us in better understanding the economic principles of aviation safety.

\section{ACKNOWLEDGMENTS}

The work of DRI is supported by the AXAICMAT Chair in Adversarial Risk Analysis, MINECO projects MTM2011-28983-C3-1 and
MTM2014-56949-C3-1-R, and the ISCH COST Action IS1304 on Expert Judgement. The work of MEC is supported by MINECO project MTM201342323-P. Started while LL was visiting Universidad Rey Juan Carlos with an AECID-PCI grant.

\section{REFERENCES}

1. ICAO. Safety Management Manual (Doc 9859 AN/474). International Civil Aviation Organization, 2013.

2. Cox LA. What's wrong with risk matrices? Risk Analysis, 2008; 28:497-512.

3. Haimes Y. Risk Modeling, Assessment, and Management. Hoboken, NJ: Wiley, 2009.

4. Jayakody D, Place S. Development of a Bayesian model to assess risk in continuing airworthiness. Presented at the 62nd Annual International Air Safety Seminar, 2009.

5. Luxhoj J. Probabilistic causal analysis for system safety risk assessments in commercial air transport. Pp. 17-38 in Proceedings of the Workshop on the Investigation and Reporting Incidents and Accidents, Williamsburg, VA, 2003.

6. Morales O. Bayesian Belief Nets and Vines in Aviation Safety and Other Applications. PhD Thesis, TU Delft, 2010.

7. IATA. The Cost of Inadvertent Slide Deployments. IATA Management Brief, 2005.

8. Bedford T, Cooke R. Probabilistic Risk Analysis: Foundations and Methods. Cambridge, UK: Cambridge University Press, 2001.

9. Bier V, Cox LA, Jr. Probabilistic risk analysis for engineered systems. Pp. 279-301 in Edwards W, von Winterfeldt D, Miles $\mathrm{R}$ (eds). Advances in Decision Analysis. New York: Cambridge University Press, 2007.

10. Cooke R. Experts in Uncertainty: Opinion and Subjective Probability in Science. New York: Oxford University Press, 1991.

11. O'Hagan A, Buck C, Daneshkhah A, Eiser R, Garthwaite $\mathrm{P}$, Jenkinson D, Oakley J, Rakow T. Uncertain Judgements: Eliciting Experts' Probabilities. Chichester, UK: Wiley, 2006.

12. Kaplan S, Garrick J. On a qualitative definition of risk. Risk Analysis, 1981; 1(1): 11-27.

13. French S, Rios Insua D. Statistical Decision Theory. London: Arnold, 2000.

14. Gelman A, Carlin J, Stern H, Rubin D. Bayesian Data Analysis. Boca Raton, FL: Chapman Hall, 2004.

15. Gelman A, Hill J. Data Analysis Using Regression and MultiLevel/Hierarchical Models. New York: Cambridge University Press, 2006

16. Rios Insua D, Ruggeri F, Wiper M. Bayesian Analysis of Stochastic Process Models. Chicester, UK: Wiley, 2012.

17. Galway L. Subjective Probability Elicitation in Cost Risk Analysis. RAND Reports, 2007.

18. Su DC, Berger J. Bayesian sequential reliability for Weibull and related distributions. Annals of the Institute of Statistical Mathematics, 1994; 46: 221-249.

19. Cook A, Tanner G. European Airline Delay Cost Reference Values. London: University of Westminster, 2011.

20. Cook A, Tanner G, Williams V, Meise G. Dynamic cost indexing. Managing airline delay costs. Journal of Air Transport Management, 2009; 15: 26-35.

21. Cook A, Tanner G, Lawes A. The hidden cost of airline unpunctuality. Journal of Transport, Economics and Policy, 2012; 46: 157-173.

22. ICAO. Annex 13 to the Convention on International Civil Aviation, Aircraft Accident and Incident Investigation. International Civil Aviation Organization, 2010.

23. FAA. The Human Factors Analysis and Classification SystemHFACS. Federal Aviation Administration, 2000. 